\title{
Gewalterfahrungen bei Patientinnen und Patienten einer Fachklinik für Alkoholabhängige: Ergebnisse einer Prävalenzerhebung und Konsequenzen für das Behandlungsprogramm
}

\author{
C. Kemmner ${ }^{1}$ \\ M. Klein ${ }^{2}$ \\ U. Zemlin
}

\author{
Experience of Violence in Patients of a Specialised Hospital for Alcohol Dependent \\ Patients of a Prevalence Study and Consequences for Treatment
}

\section{Zusammenfassung}

Die Lebenserfahrungen vieler Abhängigkeitskranker sind gekennzeichnet durch sexuellen Missbrauch und körperliche und seelische Gewalt in der Kindheit und der Adoleszenz und durch den nachfolgenden dysfunktionalen Versuch einer Konflikt- und Lebensbewältigung durch Suchtmittel. In der vorliegenden Untersuchung wurden 313 Patienten und Patientinnen, die sich im Rahmen ihrer stationären Therapie in einer Fachklinik für Alkohol- und Medikamentenabhängige befanden, mittels verschiedener Instrumente hinsichtlich ihrer Gewalterfahrungen und ihres Gewaltverhaltens befragt. 54,8\% der weiblichen und 47,7\% der männlichen Patienten haben manchmal, häufig oder sehr oft leichte Formen der körperlichen Gewalt im Laufe ihres bisherigen Lebens erfahren. Der Anteil der Frauen, die manchmal, häufig oder sehr oft schwere Formen der körperlichen Gewalt erlebt haben (26\%), ist signifikant größer als der entsprechende Anteil der Männer (15,2\%). Im Interview berichten 32,3\% der Patientinnen und 4,5\% der Patienten von sexuellem Missbrauch. 32\% der Personen mit körperlicher Gewalterfahrung in Kindheit und Jugend erlebten auch im Erwachsenenalter wieder körperliche Gewalt. 48,5\% der Personen, die in der Kindheit und Jugend körperliche Gewalt erlebt haben, übten auch selbst Gewalt aus. Die Befunde stützen die Reviktimisierungshypothese und legen nahe, dass männliche und weibliche Patienten mit Gewalterfahrungen auch selbst Gewalt ausüben. Die Ergebnisse sprechen dafür, Gewalterfahrungen und Gewaltverhalten in den Fokus der Abhängigkeitsbehandlung zu nehmen.

\section{Abstract}

Many addicts' experiences of life are marked by sexual abuse as well as physical and psychological violence in childhood and adolescence, and by the ensuing dysfunctional attempt at coping with conflict and life using addictive substances. In the study presented 313 inpatients who within the context of their therapy were currently staying in a special clinic for alcohol and substance dependence were surveyed on their violence experience and violence behavior by means of different instruments. $54.8 \%$ of female and $47.7 \%$ of male patients have sometimes, often or very often experienced forms of physical violence in the course of their lives up to now. The share of women who have sometimes, often or very often experienced severe forms of physical violence $(26 \%)$ is significantly higher than the corresponding share of men (15.2\%). In the interview $32.3 \%$ of female and $4.5 \%$ of male patients report of sexual abuse. $32 \%$ of those with experiences of physical violence in childhood and youth again experienced violence at an adult age. $48.5 \%$ of those with experiences of physical violence in childhood and youth later also used violence themselves. The results support the revictimization hypothesis and suggest that male and female patients with violence experience also use violence themselves. These findings speak in favor of focussing on violence experience and violence behavior in the treatment for dependency.

\section{Key words}

Experiencing violence $\cdot$ using violence $\cdot$ gender specific differences $\cdot$ revictimization · passing on violence $\cdot$ need for therapy

Institutsangaben

${ }^{1}$ Fachklinik Wilhelmsheim, Oppenweiler

${ }^{2}$ Katholische Fachhochschule Nordrhein-Westfalen, Köln

Korrespondenzadresse

Claudia Kemmner · Fachklinik Wilhelmsheim · 71570 Oppenweiler · E-mail: ckemmner@ahg.de

Bibliografie

Suchttherapie 2004; 5: 124-131 @ Georg Thieme Verlag KG Stuttgart · New York

DOI 10.1055/s-2004-813533

ISSN 1439-9903 
Schliusselwörter

Gewalterleben · Gewaltausübung · geschlechtsspezifische Differenzen • Reviktimisierung • Weitergabe von Gewalt • Therapiebedarf

Die Zusammenhänge zwischen Sucht und Gewalt sind komplex. So kommt es durch die alkoholbedingte Herabsetzung der Kontrollfähigkeit zu einer Zunahme aggressiver Handlungen insbesondere im innerfamiliären Bereich. Psychische, körperliche oder sexuelle Gewalterfahrungen in der Kindheit und Jugend haben eine Prädiktorfunktion bei der Vorhersage von Suchtstörungen und/oder anderen psychischen Auffälligkeiten. Weiterhin ist der Einsatz von Suchtmitteln für Frauen häufig im Sinne einer Überlebensstrategie zu sehen, d.h. es ist ein Versuch, traumatische Erlebnisse zu bewältigen [1].

\section{Gewaltverhalten bei Alkoholkonsum}

Es ist bereits seit langem bekannt, dass Alkoholkonsum und menschliches Gewaltverhalten eng zusammen hängen [2, 3]. Dabei sind im Kontext von Alkoholmissbrauch sowohl die erhöhte Wahrscheinlichkeit der Ausübung gewalttätigen Verhaltens als auch des Erleidens von Gewalt (Viktimisierung) im personalen Umfeld gut dokumentiert und deshalb auch klinisch zu berücksichtigen. Nach den Untersuchungen von Pernanen [4] sind in $48 \%$ aller Fälle von Gewaltverbrechen gegen Frauen die Täter zum Tatzeitpunkt alkoholisiert. Im National Crime Victimization Survey (NCVS) aus den Jahren 1992 und 1993 fand sich eine entsprechende Quote von 54\% [5]. Bestimmte Substanzen sind besonders häufig mit Gewaltverhalten assoziiert. Dies sind in erster Linie Amphetamine und Kokain als Psychostimulanzien sowie am häufigsten Alkohol [6]. Übermäßiger Alkoholkonsum gilt demnach zu Recht als ein gewichtiger Risikofaktor für antisoziales Verhalten [6]. Von den volkswirtschaftlichen Folgekosten durch Alkoholprobleme entfallen mindestens 11,3\% auf Gewalthandlungen unter Alkoholeinfluss und deren Folgen [7]. Sowohl für Körperverletzungs- als auch für Tötungsdelikte lässt sich aus den deutschen und internationalen Kriminalarchiven und Polizeistatistiken ein langfristiger paralleler Verlauf zwischen der Deliktfrequenz und dem Pro-Kopf-Alkoholkonsum der Gesamtbevölkerung ablesen $[8,9]$. Außerdem zeigen diese Dokumentationen fast ausnahmslos eine Zunahme der Gewaltdeliktschwere mit zunehmender Alkoholintoxikation.

Dieser Zusammenhang bestätigt sich insbesondere für Personen mit Alkoholintoxikation und alkoholbezogenen Problemen (Missbrauch und Abhängigkeit). Mit steigenden Intoxikationsraten, die zum großen Teil von Personen mit alkoholbezogenen Problemen erreicht werden, nimmt innerhalb bestimmter Bandbreiten das Risiko für Gewaltverhalten zu [8]. Der enge räumliche und zeitliche Zusammenhang zwischen Alkoholtrinken, insbesondere Alkoholintoxikation, und aggressivem Verhalten im Sinne einer Risikoerhöhung und -verdichtung konnte auch in einer Vielzahl von experimentellen Studien und Felduntersuchungen bestätigt werden $[6,10]$.
Weitgehend offen ist bis jetzt jedoch die Frage geblieben, ob Alkoholtrinken nur ein Begleitumstand von Gewaltverhalten oder eine Hauptursache darstellt. Allerdings haben sich die Hinweise vermehrt, dass Alkoholintoxikation innerhalb eines komplexen Bedingungsgefüges einen erheblichen kausalen Beitrag zur Verursachung von Gewaltverhalten zu leisten vermag [11].

\section{Funktionalität des Alkoholkonsums}

Der Einfluss psychoaktiver Substanzen auf das Gewaltverhalten kann als risikohaft auslösender und erleichternder Faktor („trigger") angesehen werden. Der Alkohol weist dabei über verschiedene Wirkkonsequenzen entscheidende Funktionalitäten zur Auslösung antisozialen Verhaltens auf. Dazu gehören die Hemmung von Ängsten, die Einschränkung der Wahrnehmung und die Stimulation psychomotorischer Funktionen [12]. Ganz offensichtlich gibt es jedoch keinen Automatismus, etwa in dem Sinne, dass erhöhter Alkoholkonsum zu erhöhter Gewaltbereitschaft führen muss. Daher betonen neuere Theorien die Wichtigkeit der zusätzlichen Berücksichtigung differenzieller Faktoren und deren Interaktionen, wie z.B. der konsumierten Alkoholmenge und -art, der neuropsychologischen Effekte des Alkohols, der sozial-kognitiven Erwartungen an die Alkoholwirkungen, zugrunde liegender oder komorbider Persönlichkeitsfaktoren und Dispositionen sowie der jeweiligen Situations- und Kontextbedingungen.

Die Analyse von Gewaltakten bei Vorliegen einer Alkoholintoxikation zeigt, dass meist ein verbaler Streit bestehend aus Drohungen und Einschüchterungen vorausgeht. Dem folgt eine mehr oder weniger schnelle Eskalation, die dann schließlich zu physischer Gewalt führen kann. Entscheidend ist oft, dass die alkoholisierte Person sich provoziert fühlt, was gerade als ein Resultat der veränderten kognitiven Funktionen anzusehen ist. Höhere Alkoholdosen gehen dabei mit einem stärker aggressiven Reagieren auf Provokationen einher [6]. Das Opfer beginnt die Auseinandersetzung genauso oft wie der alkoholintoxikierte Täter. Allerdings ist es die alkoholintoxikierte Person, die häufiger eine Eskalation der Auseinandersetzung beschleunigt [13].

\section{Täter und Opfer}

In der alkohol- und drogenbezogenen Forschungsliteratur dominieren nach wie vor Studien, die auf die Ausübung des Gewaltverhaltens fokussieren. Der Aspekt der Viktimisierung wurde in den umfangreichen Arbeiten von Pernanen [4] erstmals aufgegriffen. In einer repräsentativen Bevölkerungsstudie mit 933 Personen ab dem 20. Lebensjahr in Thunder Bay (Ontario) fand sich für Gewaltverhalten eine Alkoholisierungsquote von 51\% für die Täter und interessanterweise auch von $30 \%$ für die Opfer [4]. Insbesondere fand sich, dass Gewaltverhalten gegenüber Frauen in einem höheren Ausmaß (39\%) von alkoholisierten Tätern im Vergleich zu Gewaltverhalten gegenüber Männern (23\%) auftrat. Bei ausschließlicher Betrachtung häuslicher Gewalt fand sich eine Alkoholisierungsquote von 39\% für die Täter und von $22 \%$ für die Opfer. Schließlich fand sich bei ausschließlicher Betrachtung von Gewalthandlungen zwischen Partnern eine Alko- 
holisierungsquote von $44 \%$ für die Täter und von $14 \%$ für die Opfer, während in $12 \%$ aller Fälle sowohl Täter als auch Opfer unter Alkoholeinfluss standen. In der nationalen Viktimisierungsstudie aus den USA [5] konnte eruiert werden, dass Alkoholintoxikationen die Wahrscheinlichkeit von Gewalthandlungen männlicher Partner gegenüber ihren Partnerinnen im Konfliktfall deutlich erhöhten.

Die Ergebnisse der national-repräsentativen Umfrage zu innerfamiliärer Partnergewalt in den USA von 1985 [14] ergaben, dass $17 \%$ der Paare in den letzten 12 Monaten mindestens einmal Gewalt durch den Partner erlebt haben, wobei Frauen sogar etwas häufiger als Täterinnen in Erscheinung traten als Männer. Ein ähnliches Ergebnis fanden Caetano et al. [15] im Rahmen einer nationalen Studie. Hier berichteten, in Abhängigkeit von der Zugehörigkeit zu einer ethnischen Gruppe, zwischen 11,5 und 23\% der Frauen, Gewalt durch ihre Partner erlebt zu haben. Zwischen 15 und 30\% der Männer berichteten von Gewalterfahrungen durch ihre Partnerinnen. 30 bis $40 \%$ der Männer und 27 bis $34 \%$ der Frauen hatten zum Zeitpunkt des Gewaltausübens Alkohol getrunken.

In Bezug auf Kinder alkoholabhängiger Eltern ist bekannt, dass diese in erhöhtem Ausmaß physischer Gewalt ausgesetzt sind. In einer Studie mit 207 jungen Erwachsenen aus alkoholbelasteten Familien und 221 unbelasteten Kontrollprobanden berichteten $13,4 \%$ der ersten Gruppe im Unterschied zu 2,3\% der zweiten Gruppe von täglicher Gewalt in der Familie [16]. Zu dem Ergebnis, dass pathologischer Substanzkonsum bei Eltern mit einem erhöhten Risiko für Vernachlässigung, Misshandlung und Missbrauch bei deren Kindern verknüpft ist, kamen auch Rose et al. [17]. Sie ermittelten für Kinder aus Alkoholikerfamilien ein erhöhtes Risiko für Inzest (3fach), körperliche Misshandlung (2fach) und für beide Arten der Misshandlung (2fach) im Vergleich zu Nichtalkoholikerfamilien.

Zahlreiche Untersuchen weisen darauf hin, dass die Wahrscheinlichkeit für Frauen, die in der Kindheit Gewalt erlebt haben, als Erwachsene erneut Opfer von innerfamiliärer Gewalt zu werden, deutlich erhöht ist [18-20]. Es gibt einige Studien, die in die Richtung weisen, dass frühe Gewalterfahrungen besonders das Selbstkonzept, den Selbstwert, das Konzept der erlernten Hilflosigkeit, den Attributionsstil, auch gerade bezüglich der erlebten Gewalt sowie die Copingstrategien beeinträchtigen.

Auch in späteren Lebensabschnitten sind Personen mit substanzbezogenen Störungen in weit höherem Maße als die Allgemeinbevölkerung traumatischen Erfahrungen ausgesetzt. Große epidemiologische Untersuchungen kamen übereinstimmend zu dem Ergebnis, dass Suchtpatienten eine etwa 1,5fach erhöhte Wahrscheinlichkeit aufweisen, traumatischen Erlebnissen wie sexueller oder körperlicher Gewalt ausgesetzt zu sein $[21,22]$.

Fragestellungen der vorliegenden Untersuchung

Vor dem Hintergrund der Entwicklung und Implementierung geeigneter, empirisch begründbarer und geschlechtsspezifischer Behandlungsformen während einer stationären Entwöhnung untersuchte die vorliegende Studie sowohl die Gewalterfahrungen als auch das Gewaltverhalten von Abhängigkeitskranken. Der Untersuchung liegen folgende Fragestellungen zugrunde:

Wie häufig werden Gewalterfahrungen und Gewaltausübung von alkohol- und medikamentenabhängigen Patientinnen und Patienten der Fachklinik berichtet?

1. Wer übte körperliche Gewalt an Patientinnen und Patienten aus?

2. Werden Personen, die in Kindheit und Jugend Gewalterfahrungen gemacht haben, als Erwachsene erneut zum Opfer (Reviktimisierung)?

3. Wie häufig üben Patientinnen und Patienten Gewalt aus und an wem?

4. Gibt es eine erhöhte Bereitschaft zur Weitergabe von Gewalt bei Personen mit eigenen Gewalterfahrungen in Kindheit und Jugend?

5. Wie bewerten Patientinnen und Patienten und Therapeuten die Gewalterfahrungen?

\section{Methoden}

\section{Stichprobe}

In die Untersuchung gingen konsekutiv alle Personen ein, die im Jahr 2002 in der Fachklinik Wilhelmsheim aufgenommen wurden, bis eine ausreichende Stichprobengröße mit gleichen Anteilen von Männern und Frauen erreicht wurde (konsekutive Totalerhebung). Aufgrund des deutlich größeren Anteils von Männern in der Klientel der Fachklinik erstreckte sich die Datenerhebung bei den Männern über drei Aufnahmemonate, bei den Frauen über 9 Monate. Eine Selektion innerhalb des Aufnahmezeitraums fand nicht statt. Es konnten 313 Patienten untersucht werden, davon waren 155 Männer und 158 Frauen.

\section{Erhebungsinstrumente}

Die Gewalterfahrungen der Patienten sowohl als Opfer als auch als Täter wurden mit einem eigens entwickelten standardisierten Interviewverfahren zur „Erfassung von Gewalterfahrungen“ (EGE) erhoben ${ }^{1}$ [23]. Das EGE-Interview bildet Gewalterfahrung in den Bereichen körperliche, psychische und sexuelle Gewalt ab. Beispielsweise wird körperliche Gewalterfahrung mit folgender Eingangsfrage erhoben: „Haben Sie in irgendwelcher Form körperliche Gewalt erlebt? Sind Sie z.B. geschlagen oder getreten worden, mit Gegenständen verletzt worden, an einzelnen Körperstellen verbrüht oder verbrannt worden?" Mit analogen Formulierungen und Beispielen wird auch psychische und sexuelle Gewalt erfragt. Erhoben wird für jeden der drei Bereiche, durch wen, wann und in welcher Häufigkeit Gewalt zugefügt wurde, ob dies die Patientinnen und Patienten heute noch beschäftigt und ob die Gewalterfahrungen nach Ansicht der Patienten und ihrer Therapeuten die Lebensentwicklung geprägt haben.

Weiterhin wird in derselben Systematik erfasst, ob die Patientinnen und Patienten selbst körperliche, psychische und sexuelle

\footnotetext{
1 Wir danken Prof. Brenda Miller und Dr. Rich Mancuso, Center for Research on Urban Social Work Practice, School of Social Work, University at Buffalo, für hilfreiche Hinweise.
} 
Gewalt („Ich habe schon mal jemanden sexuell belästigt, zu sexuellen Handlungen gezwungen, vergewaltigt“) ausgeübt haben (an wem, wann und in welcher Häufigkeit) und wie sehr sie dies heute noch beschäftigt. Abschließend schätzen die Therapeuten die Notwendigkeit der therapeutischen Bearbeitung der Gewaltthematik ein. Das Interview wird durch die Bezugstherapeuten routinemäßig bei allen Patienten und Patientinnen in der dritten Woche nach Aufnahme durchgeführt. Zu diesem Zeitpunkt liegen in der Regel die Ergebnisse der Anamnesen und der sonstigen psychologischen Testungen bereits vor. Die Ergebnisse des Interviews fließen in die Behandlungsplanung ein.

Im Sinne einer Validierung des Instruments und um weitere Daten zu Konfliktlösestrategien zu erhalten, wurden als zweites zentrales Instrument die „Conflict Tactics Scales“ (CTS) von Straus [24] in der deutschen Übersetzung von Klein [25] in der deutschen Fassung eingesetzt. Es erfragt differenziert Gewalterfahrungen in Kindheit und Jugend (bis 18 Jahre) sowie im Erwachsenenalter (über 18 Jahre), wobei hauptsächlich auf den Bereich der körperlichen Gewalt fokussiert wird, ergänzt durch Fragen zu sexuellem Missbrauch und zu Vernachlässigung. Die CTS erlauben eine Differenzierung in „leichte Gewalt“ (= Züchtigung: Ihnen eine runtergehauen, Sie hart angepackt oder gestoßen, mit Gegenstand nach Ihnen geworfen, Sie mit Gegenstand geschlagen) und „schwere Gewalt“ (= Misshandlung: Sie mit der Faust geschlagen, Sie geprügelt oder zusammengeschlagen, Sie gewürgt, Sie mit einer Waffe bedroht, Ihnen Verbrennungen zugefügt, eine Waffe gegen Sie eingesetzt) sowie eine Abstufung der Häufigkeit entsprechender Gewalterfahrungen bzw. -ausübungen auf der Skala „nie - einmal - selten - manchmal - häufig - sehr oft“. Aus diesen Kategorien wurden zwei dichotome Variablen gebildet. Die eine Variable stellt die Ausprägung „Gewalterfahrungen vorhanden“ der Ausprägung „keine Gewalterfahrungen vorhanden“ gegenüber. In der zweiten Variablen wurden die Kategorien „nie - einmal - selten“ zusammengefasst und die Kategorien „manchmal - häufig - sehr oft“ zur Ausprägung „häufiger als selten“ zusammengefasst.

Soziodemografische Merkmale der Stichprobe wurden der Basisdokumentation entnommen.

\section{Statistik}

Die statistische Auswertung wurde mit SPSS 10.0 für Windows durchgeführt. Zur Überprüfung der Bedeutsamkeit der Häufigkeitsangaben wurde als statistisches Verfahren der $\chi 2$-Test angewandt.

\section{Ergebnisse}

Die Zusammensetzung der Untersuchungsstichprobe nach soziodemografischen Merkmalen beschreibt Tab. 1. 23,5\% der Männer und 25,9\% der Frauen (n. s.) gaben in der Anamnese Suchtprobleme des Vaters an. Von Suchtproblemen der Mutter berichteten 6,5\% der Männer und 12,9\% der Frauen (n. s.). 20,9\% der Frauen, aber nur 6,5\% der Männer berichteten von Suchtproblemen bei ihrem Partner bzw. ihrer Partnerin $\left(\chi^{2}=13,76, \mathrm{df}=1\right.$, $\mathrm{p}=0,000)$. Die Stichprobenzusammensetzung entspricht dem Gesamtkollektiv des Behandlungsjahrgangs 2002 der Fachklinik [26] und kann daher, abgesehen von der Geschlechterverteilung, als repräsentativ für die Klientel der Fachklinik angesehen werden.

Tab. 2 vergleicht die prozentuale Häufigkeit von körperlichen Gewalterfahrungen der Fachklinikstichprobe, die „häufiger als selten“ erlebt wurden, mit den Daten einer repräsentativen Stichprobe aus der deutschen Bevölkerung [19]. Beide Stichproben wurden mittels der CTS befragt. Während sich bei leichter Gewalt nur ein geringer Unterschied abzeichnet, sind die Unterschiede bei schwerer Gewalt augenfällig: Der Prozentsatz von Personen, die häufiger als selten schwere körperliche Gewalterfahrungen machten, ist unter den Patientinnen und Patienten der Fachklinik fast dreimal so hoch wie in der Normalbevölkerung.

Bezogen auf die gesamte bisherige Lebenszeit zeigte sich in Bezug auf die Häufigkeit von körperlichen Züchtigungen kein signifikanter Geschlechtsunterschied $(\chi 2=1,55, \mathrm{df}=1 ; \mathrm{p}=0,256)$. Patientinnen und Patienten der Fachklinik gaben in den CTS mit 54,8 zu 47,4\% vergleichbar hohe Raten der Skalenausprägung „häufiger als selten“ an. Die Prävalenz von Misshandlung ist hin-

\begin{tabular}{|c|c|c|c|c|}
\hline Variable & Level & $\begin{array}{l}\text { Gesamtstichprobe } \\
n=313\end{array}$ & $\begin{array}{l}\text { Männer } \\
n=155\end{array}$ & $\begin{array}{l}\text { Frauen } \\
n=158\end{array}$ \\
\hline \multirow[t]{2}{*}{ Geschlecht } & männlich & $49,5 \%$ & $100 \%$ & - \\
\hline & weiblich & $50,5 \%$ & - & $100 \%$ \\
\hline Alter & Mittelwert & 45,9 & 44,9 & 46,7 \\
\hline \multirow[t]{3}{*}{ Erwerbsstatus } & erwerbstätig & $52,4 \%$ & $51,0 \%$ & $53,8 \%$ \\
\hline & arbeitslos & $35,5 \%$ & $44,5 \%$ & $26,6 \%$ \\
\hline & sonstige & $12,1 \%$ & $4,5 \%$ & $19,6 \%$ \\
\hline \multirow[t]{3}{*}{ Schule } & $\begin{array}{l}\text { Hauptschule und } \\
\text { niedriger }\end{array}$ & $58,5 \%$ & $63,3 \%$ & $53,8 \%$ \\
\hline & $\begin{array}{l}\text { Realschule/Abitur/ } \\
\text { Hochschule }\end{array}$ & $41,2 \%$ & $36,7 \%$ & $35,4 \%$ \\
\hline & anderer Abschluss & $0,3 \%$ & - & - \\
\hline \multirow[t]{3}{*}{ Partnerbeziehung } & allein stehend & $35,1 \%$ & $43,2 \%$ & $27,2 \%$ \\
\hline & zeitweilige Beziehung & $5,1 \%$ & $4,5 \%$ & $5,7 \%$ \\
\hline & feste Beziehung & $39,7 \%$ & $52,3 \%$ & $67,1 \%$ \\
\hline
\end{tabular}

Tab. 1 Soziodemographische Merkmale (Basisdokumentationsdaten) 


\begin{tabular}{llc}
\hline $\begin{array}{l}\text { Gewalterfahrungen in Kindheit und Jugend } \\
\text { (Conflict Tactics Scale (CTS) „häufiger als selten“) }\end{array}$ & $\begin{array}{l}\text { eigene Erhebung } \\
\text { (\%) }\end{array}$ & $\begin{array}{l}\text { Wetzels } 1997 \\
\text { (\%) }\end{array}$ \\
\hline leichte Gewalt/körperliche Züchtigung & 46,3 & 38,4 \\
- Ihnen eine runtergehauen & 41,9 & 36,5 \\
- Sie hart angepackt oder gestoßen & 25,2 & 12,1 \\
- mit Gegenstand nach Ihnen geworfen & 8,6 & 3,7 \\
- Sie mit einem Gegenstand geschlagen & 22,7 & 4,6 \\
schwere Gewalt/körperliche Misshandlung & 13,7 & 4,7 \\
- Sie mit der Faust geschlagen & 11,2 & 2,6 \\
- Sie geprügelt, zusammengeschlagen & 8,3 & 3,5 \\
- Sie gewürgt & 1,3 & 0,7 \\
- Sie mit einer Waffe bedroht & 1,0 & 0,4 \\
- Ihnen Verbrennungen zugefügt & 0,6 & 0,4 \\
- eine Waffe gegen Sie eingesetzt & 0,6 & 0,3 \\
\hline
\end{tabular}

Tab. 2 Gewalterfahrungen in Kindheit und Jugend in der Stichprobe der Fachklinik Wilhelmsheim $(n=313)$ im Vergleich mit einer Repräsentativerhebung (Wetzels, 1997; $\mathrm{n}=3249$ )

gegen bei Frauen mit 26\% signifikant höher als bei Männern $(15,2 \%)(\chi 2=5,51, \mathrm{df}=1, \mathrm{p}=0,23)$.

Bezogen auf Gewalterfahrungen während des Erwachsenenlebens zeigen sich signifikante Unterschiede zwischen Männern und Frauen sowohl bei körperlichen Züchtigungen als auch bei Misshandlungen (vgl. Abb.1). Von den Frauen, die im Erwachsenenalter "häufiger als selten“ Gewalt erfahren haben, leben 39,5\% mit einem suchtmittelabhängigen Partner zusammen, während von den Frauen, die gar keine oder nur selten Gewalt erlebt haben, nur 14,2\% mit einem Partner mit Suchtproblemen zusammenleben $(\chi 2=12,03, \mathrm{df}=1, \mathrm{p}=0,001)$.

In den EGE-Interviews machten 98 Männer und 106 Frauen Angaben zu körperlichen Gewalterfahrungen. Väter, bzw. Stiefväter, stehen als Täter sowohl bei den befragten Männern (56,1\%) als auch bei den Frauen (42,5\%) im Vordergrund, gefolgt von Müttern bzw. Stiefmüttern, die bei Männern $(41,8 \%)$ und Frauen (42,5\%) etwa gleichhäufig körperliche Gewalt ausübten. Frauen geben darüber hinaus häufiger als Männer den Partner als Täter an (56,6 zu 6,1\%). Männer sind insgesamt häufiger als Frauen Opfer außerfamiliärer Gewalt, z.B. durch Fremde (28,6 zu 3,8\%). Im

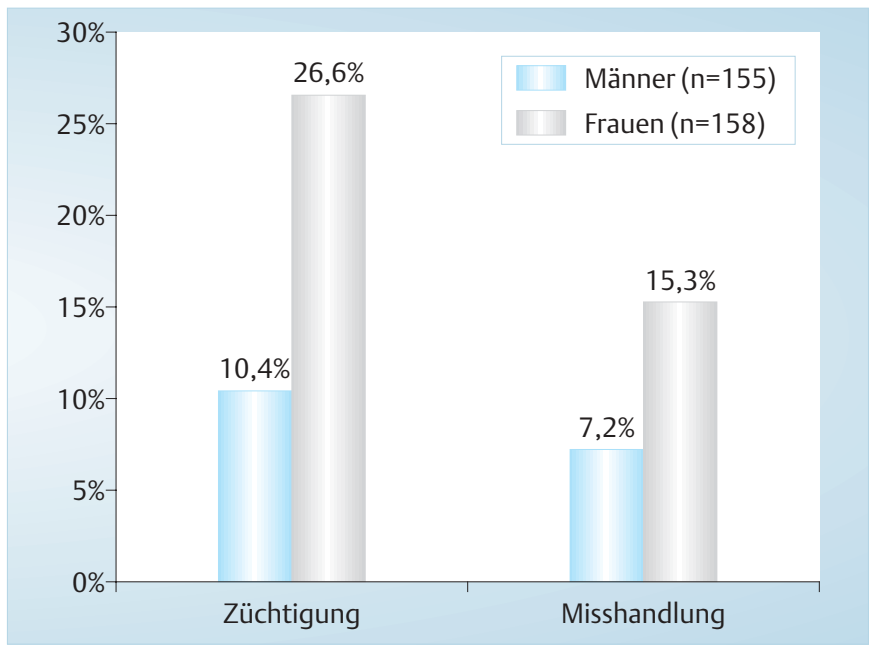

Abb. 1 Gewalterfahrung im Erwachsenenalter nach Geschlecht: körperliche Züchtigung, $\chi 2=13,51, \mathrm{df}=1, \mathrm{p}=0,000$; körperliche Misshandlung, $\chi 2=5,07, d f=1, p=0,018)$, CTS, Skalenausprägung „häufiger als selten“.

EGE-Interview wurde eine Lebenszeitprävalenz für sexuelle Gewalterfahrungen von 4,5\% bei Männern und 32,3\% bei Frauen ermittelt. Der Unterschied ist hochsignifikant $(\chi 2=39,96, \mathrm{df}=1$, $\mathrm{p}=0,000$ ). 39,6\% der Frauen, die im EGE-Interview körperliche Gewalterfahrung angeben, haben auch sexuelle Gewalt erfahren $(\chi 2=7,95, \mathrm{df}=1, \mathrm{p}=0,006)$, bei den Männern waren es hingegen nur $6,1 \%$ (n. s.).

$32 \%$ der Personen mit „häufiger als selten“ erlebter körperlicher Gewalterfahrung in Kindheit und Jugend erfahren auch im Erwachsenenalter wieder „häufiger als selten“ körperliche Gewalt. Im Vergleich dazu gilt dies nur für $8 \%$ der Personen, die körperliche Gewalt in Kindheit und Jugend nicht bzw. selten erlebt hatten. Die Reviktimisierung ist bei Frauen deutlicher ausgeprägt als bei Männern (vgl. Tab. 3).

Wie aus Tab. 4 ersichtlich wird, geben ca. zwei Drittel der Gesamtstichprobe im EGE-Interview körperliche Gewalterfahrung an. Davon übten $48,5 \%$ auch selbst körperliche Gewalt aus. Von den Personen, die körperliche Gewalt nicht selbst erlebt hatten, waren es nur 9,2\%. Der Unterschied ist hochsignifikant. Bei Männern ist der Zusammenhang von Gewalterfahrung und Gewaltausübung noch deutlicher als bei Frauen.

Bezogen auf die Fragen, an wem körperliche Gewalt ausgeübt wird, zeigt sich in den EGE-Interviews ein geschlechtsspezifischer Unterschied. Männer üben Gewalt überwiegend an Fremden $(49,3 \%)$ aus, gefolgt von Bekannten $(31,3 \%)$ und den Partnerinnen (23,9\%). Frauen geben an, gewalttätige Handlungen überwiegend an ihren Kindern (53,5\%) und ihren Partnern $(32,6 \%)$ zu begehen.

Die Gewaltthematik ist für Frauen und Männer in ihrem aktuellen Erleben unterschiedlich häufig präsent. 30,5\% der Frauen mit Gewalterfahrung geben an, dass diese sie heute noch häufig beschäftige (Männer: 9,3\%), während Männer zu 52,6\% angeben, sich gar nicht mehr damit zu befassen (vgl. Abb. 2). Mehr Männer als Frauen gaben an, in ihrer Entwicklung gar nicht von der Gewalterfahrung geprägt zu sein (42,7 zu 34,6\%), während mehr Frauen angaben, dass ihre Entwicklung sehr von der Gewalterfahrung geprägt worden sei (28,8 zu $12,5 \%)$. 


\begin{tabular}{|c|c|c|c|c|}
\hline \multirow[t]{2}{*}{ Stichprobe } & \multicolumn{2}{|c|}{$\begin{array}{l}\text { körperliche Gewalterfahrung in Kindheit und } \\
\text { Jugend }\end{array}$} & \multirow[t]{2}{*}{ Signifikanz } & \multirow[t]{2}{*}{$\chi^{2}$} \\
\hline & ja & nein & & \\
\hline \multirow[t]{3}{*}{ gesamt } & $147(47,6 \%)$ & $162(52,4 \%)$ & n. s. & - \\
\hline & \multicolumn{4}{|c|}{ davon körperliche Gewalt als Erwachsene erfahren: } \\
\hline & $47(32 \%)$ & $13(8 \%)$ & $p=, 000$ & 28,25 \\
\hline \multirow[t]{3}{*}{ Männer } & $71(46,4 \%)$ & $82(53,6 \%)$ & n.s. & - \\
\hline & \multicolumn{4}{|c|}{ davon körperliche Gewalt als Erwachsene erfahren: } \\
\hline & $15(21,1 \%)$ & $3(3,7 \%)$ & $\mathrm{p}=, 01$ & 11,19 \\
\hline \multirow[t]{3}{*}{ Frauen } & $76(48,7 \%)$ & $80(51,3 \%)$ & n. s. & - \\
\hline & \multicolumn{4}{|c|}{ davon körperliche Gewalt als Erwachsene erfahren: } \\
\hline & $32(42,1 \%)$ & $10(12,5 \%)$ & $p=, 00$ & 17,36 \\
\hline
\end{tabular}

\begin{tabular}{|c|c|c|c|c|}
\hline Stichprobe & $\begin{array}{l}\text { körperliche Gewalt- } \\
\text { erfahrung in Kindheit } \\
\text { und Jugend? } \\
\text { ja }\end{array}$ & $\begin{array}{l}\text { körperliche Gewalt- } \\
\text { erfahrung in Kindheit } \\
\text { und Jugend? } \\
\text { nein }\end{array}$ & Signifikanz & $\chi^{2}$ \\
\hline \multirow[t]{3}{*}{ gesamt } & $204(65,2 \%)$ & $109(34,8 \%)$ & & \\
\hline & \multicolumn{4}{|c|}{ davon körperliche Gewalt ausgeübt: } \\
\hline & $48,5 \%$ & $9,2 \%$ & $p=, 00$ & 48,48 \\
\hline \multirow[t]{3}{*}{ Männer } & $98(63,2 \%)$ & $57(36,8 \%)$ & & \\
\hline & \multicolumn{4}{|c|}{ davon körperliche Gewalt ausgeübt: } \\
\hline & $63,3 \%$ & $8,8 \%$ & $p=, 000$ & 43,61 \\
\hline \multirow[t]{3}{*}{ Frauen } & $106(67,1 \%)$ & $52(32,9 \%)$ & & \\
\hline & \multicolumn{4}{|c|}{ davon körperliche Gewalt ausgeübt: } \\
\hline & $34,9 \%$ & $9,6 \%$ & $p=, 001$ & 11,43 \\
\hline
\end{tabular}

Tab. 3 Reviktimisierung: körperliche Gewalterfahrung in Kindheit und Jugend sowie im Erwachsenenalter; CTS, Skalenausprägung „häufiger als selten“
Tab. 4 Körperliche Gewalterfahrung und Ausübung körperlicher Gewalt: EGE, Skalenausprägung „häufiger als selten“

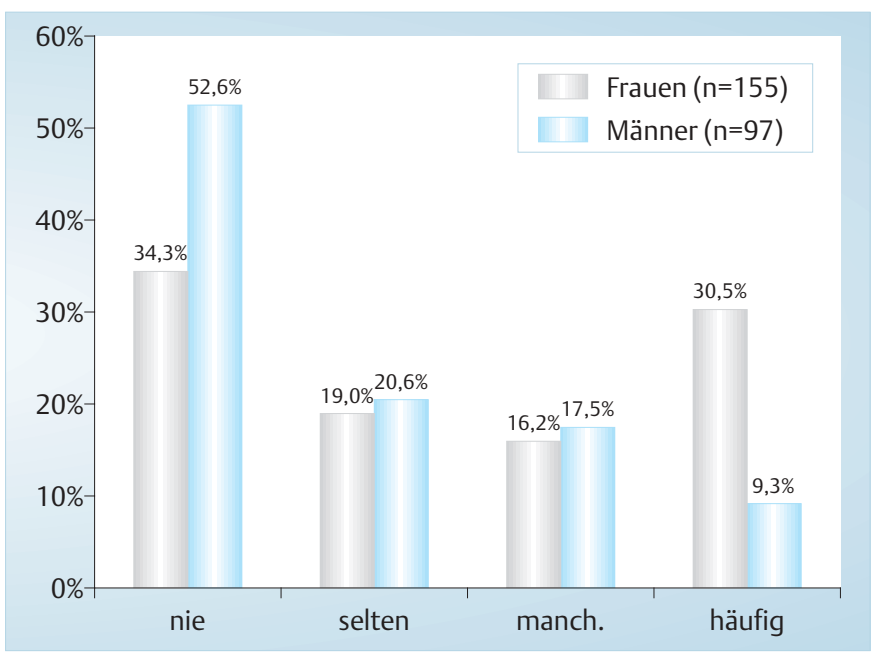

Abb. 2 Bewertung der körperlichen Gewaltproblematik durch Patienten und Patientinnen (EGE). Wie oft beschäftigt Sie die Gewalterfahrung heute noch?; $\mathrm{n}=212$.

Die Therapeutinnen und Therapeuten der Fachklinik sehen bei 46,2\% der Frauen, jedoch nur bei 24,5\% der Männer einen Therapiebedarf für die Gewaltproblematik. Letztere umfasst sowohl Gewalterfahrungen als auch die Ausübung von Gewalt. Werden in die Auswertung nur die Patienten einbezogen, die Gewalterfahrung angeben oder Gewalt ausgeübt haben, dann wird bei 57,1\% der Frauen und bei 35,6\% der Männer ein weiterer Therapiebedarf konstatiert.

\section{Diskussion}

In der vorliegenden Untersuchung haben $54,8 \%$ der weiblichen und $47,7 \%$ der männlichen Patienten häufiger als selten leichte Formen der körperlichen Gewalt im Laufe ihres bisherigen Lebens erfahren. Signifikant mehr Frauen (26\%) als Männer (15,2\%) haben häufiger als selten schwere Formen der körperlichen Gewalt erlebt. Im Interview berichten 32,3\% der Patientinnen und 4,5\% der Patienten von sexuellem Missbrauch. Verglichen mit anderen klinischen Stichproben an Suchtpatientinnen, liegen diese Zahlen in einer ähnlichen Größenordnung, wobei die männlichen Patienten unserer Studie seltener über sexuellen Missbrauch berichten [27, 28]. 32\% der Personen mit körperlicher Gewalterfahrung in Kindheit und Jugend erlebten auch im Erwachsenenalter wieder körperliche Gewalt. 48,5\% der Personen, die in der Kindheit und Jugend körperliche Gewalt erlebt haben, übten auch selbst Gewalt aus.

Bei der Betrachtung der Ergebnisse im Einzelnen fällt zunächst auf, dass ein großer Teil der Patienten und Patientinnen körperliche, psychische und/oder sexuelle Gewalt erlebt und/oder ausgeübt hat. Schaut man auf die Elterngeneration der Patientinnen und Patienten, fällt auf, dass bei beiden die Häufigkeit einer Suchtmittelabhängigkeit eines oder beider Elternteile deutlich höher ist als in der Normalbevölkerung. So berichten 25,8 \% der Patientinnen (23,9\% der Patienten) von Suchtproblemen beim Vater, $12 \%$ der Patientinnen (6,5\% der Patienten) von Suchtproblemen bei der Mutter. Dies entspricht den Ergebnissen aus der Literatur, dass das Risiko, Suchtprobleme oder andere psy- 
chische Auffälligkeiten zu entwickeln, für Kinder aus suchtbelasteten Familien deutlich erhöht ist [17, 18, 29].

Gravierende Unterschiede gibt es beim Erleben körperlicher Gewalt im Erwachsenenalter. Hier geben signifikant mehr Frauen als Männer an, leichtere körperliche Gewalt (Züchtigung) und schwere körperliche Gewalt (Misshandlungen) erlebt zu haben, und zwar fast ausschließlich durch ihre Partner ( 56 versus 6,1\%). Es ist anzunehmen, dass dieses Ergebnis auch im Zusammenhang mit den Angaben zu sehen ist, dass 20,9\% unserer Patientinnen, aber nur 6,5\% unserer Patienten einen Partner mit Suchtproblemen haben. Von den Frauen, die im Erwachsenalter Gewalt erlebt haben, haben knapp 40\% einen suchtmittelabhängigen Partner, während von den Frauen, die keine Gewalt im Erwachsenenalter erlebt haben, nur ca. 14\% einen suchtmittelabhängigen Partner haben. Das Ergebnis, dass nur sehr wenige der männlichen Patienten angeben, Gewalt durch ihre Partnerin erlebt zu haben, ist auf dem Hintergrund, dass Frauen genau so oft wie Männer in ihren Partnerschaften gewalttätig werden, bemerkenswert. Die Zahlen der vorliegenden Untersuchung, dass Frauen ähnlich häufig wie Männer ihren Partnern gegenüber Gewalt ausüben, stimmen mit den Befunden der national-repräsentativen Umfrage von Straus [14] und der von Caetano [15] überein. Möglicherweise sind die Angaben der Männer durch Scham und Unverträglichkeit mit dem Männerbild verzerrt. Eine andere Erklärung könnte sein, dass die alkoholabhängigen Männer deutlich seltener eine suchtmittelabhängige und damit potenziell gewalttätige Partnerin haben. Ein weiterer Faktor könnten geschlechtsspezifisch unterschiedliche Konsequenzen auf das Ansprechen von Gewalt durch den Partner sein. So erhalten Frauen nach Berichten von Gewalterfahrungen eher Aufmerksamkeit und Unterstützung als Männer. Männer hingegen müssen eher mit Abwertungen, Schuldzuweisungen und Unglauben und letztendlich Ausgrenzung rechnen, da es den gängigen Täter-Opfer-Klischees widerspricht. Auch gibt es mittlerweile ein breites frauenspezifisches, aber kaum ein männerspezifisches Angebot (Frauenberatungsstellen, Frauenhäuser, spezifische Therapieangebote etc.).

Gewalt in der Kindheit erhöht das Risiko erneuter Viktimisierung im Erwachsenenalter [19]. In der vorliegenden Untersuchung zeigt sich bei den männlichen Patienten eine Reviktimisierung (21,1\%), die allerdings bei den Frauen fast doppelt so häufig ist $(42,1 \%)$.

Es gibt Hinweise darauf, dass nicht so sehr einzelne isolierte Gewalterfahrungen in der Kindheit, sondern insbesondere das $\mathrm{Zu}$ sammenwirken der Wahrnehmung elterlicher Partnergewalt und die eigene Viktimisierungserfahrung zu einer Erhöhung des Risikos führen, dass Frauen als Erwachsene in ihrer Familie erneut Opfer schwerer Gewalt werden $[20,30]$.

Das Ergebnis, dass Frauen, die über sexuelle Gewalterfahrungen berichten, in der Regel auch körperliche Gewalt erlebt haben, stimmt mit anderen Untersuchungsergebnissen überein [31]. Obwohl es klare Hinweise gibt, dass körperliche und/oder psychische Misshandlungen in der Kindheit ähnliche Auswirkungen bezüglich der Entwicklung einer Suchtmittelabhängigkeit oder sonstiger psychischer Auffälligkeiten haben [32, 33], bezieht sich der Großteil der Untersuchungen auf sexuellen Missbrauch. Hier sehen wir weiteren Forschungsbedarf.
Bei der Betrachtung der Ergebnisse zum Gewaltverhalten zeigt sich, dass 63,3\% der Männer, die in der Kindheit und Jugend körperliche Gewalt erlebt haben, auch selbst Gewalt ausgeübt haben, bei den Frauen sind es 34,9\%. Für beide Geschlechter ist mittlerweile nachgewiesen, dass Eltern, die in ihrer Kindheit Gewalt erlebt haben, signifikant häufiger aktive körperliche Erziehungsgewalt ausüben als Eltern ohne entsprechende Erfahrungen [34]. Als Hintergrund sind Modelllernen und mangelnde Vermittlung adäquater Konfliktlösungsstrategien zu nennen. Während Frauen vor allem Gewalt innerhalb der Familie, d.h. primär ihren Kindern und ihren Partnern gegenüber anwenden, üben Männer am häufigsten außerfamiliären Personen gegenüber Gewalt aus. Das mag darin begründet sein, dass Mütter in der Regel deutlich mehr Zeit mit ihren Kindern verbringen, da die Erziehung meistens durch sie geleistet wird.

Ein geschlechtsspezifischer Unterschied bildet sich auch in den Ergebnissen der erlebten Beeinträchtigung durch die Gewalterfahrungen männlicher und weiblicher Patienten in der Entwicklung und in der aktuellen Lebenssituation ab. In beiden Punkten gaben Frauen eine signifikant höhere Belastung an. Wahrscheinlich sind hier verschiedene Faktoren von Bedeutung. Zum einen berichten Frauen häufiger über psychische Belastungen, was im Zusammenhang mit einer höheren Selbstwahrnehmungsfähigkeit stehen könnte. Bei Männern ist davon auszugehen, dass sie eher sozial erwünscht im Sinne der rollenspezifischen Erwartung antworten. Männer attribuieren negative Ereignisse und Misserfolge eher external und werden durch die gesellschaftliche Rollenerwartung darin bestärkt, Ärgerreaktionen zu zeigen. Zum anderen ist die zeitliche Nähe zu Gewalterfahrungen bei den Frauen viel enger, da mehr Frauen, primär durch ihre Partner, im Erwachsenenalter Gewalt erlebt haben.

Interessant ist an dieser Stelle, dass sich bei der Einschätzung des Therapiebedarfs für die Gewaltproblematik durch die Therapeuten und Therapeutinnen der Fachklinik ein ähnlich großer, signifikanter geschlechtsspezifischer Unterschied zeigt. Hier bieten sich verschiedene Interpretationsmöglichkeiten an. So könnten sich die Therapeuten überwiegend an den Aussagen der Patientinnen und Patienten bezüglich des Therapiebedarfs orientiert haben oder sie haben sich in ihrer Einschätzung von weiteren Befunden leiten lassen. Möglich wäre auch, dass sie ähnlich wie die weiblichen und männlichen Patienten aufgrund traditioneller Rollenerwartungen Frauen eher einen therapeutischen Bedarf zugestanden haben.

Die Häufigkeit der Befunde in CTS und EGE zu körperlicher, psychischer und sexueller Gewalt sprechen dafür, dass das Interviewverfahren EGE Gewalt sensibler erfasst als der Fragebogen CTS. Die CTS erfassen andererseits die Art und Häufigkeit der körperlichen Gewalterfahrungen differenzierter, sexuelle Gewalterfahrungen hingegen nur global mit einer Frage. Um die Vorteile der beiden Verfahren zu integrieren, wurde der EGE überarbeitet und sowohl bei der Erhebung der Gewalterfahrungen wie auch der Gewalthandlungen Fragen nach der Häufigkeit und nach der Art der Gewalt ergänzt. Da unsere klinischen Beobachtungen wie auch die Ergebnisse von Wetzels dafür sprechen, dass auch die Wahrnehmung elterlicher Partnergewalt in der Herkunftsfamilie das Risiko einer innerfamiliären Viktimisie- 
rung im Erwachsenenalter erhöht, wurden entsprechende Fragen in die revidierte Form des EGE aufgenommen.

\section{Therapeutische Implikationen}

Es ist deutlich geworden, wie wichtig das Thema Gewalt mit seinen vielen Facetten in der Behandlung von suchtmittelabhängigen Menschen ist. Daraus ergeben sich Konsequenzen für die Behandlung. So ist es Aufgabe einer Suchtklinik, einen Rahmen zu bieten, der es Patienten und Patientinnen ermöglicht, sich frühzeitig mit der Thematik Gewalterleben/-Gewaltausübung auseinander setzen zu können. Eine effektivere Bearbeitung von Gewaltproblemen bei Suchtmittelabhängigen verbessert die Chance, dass weniger Personen im Umfeld - insbesondere Partner und Kinder schwere Traumatisierungen erleiden, die sie wiederum besonders vulnerabel für Suchtstörungen machen. Die Untersuchung weist erneut auf die Notwendigkeit hin, die Dichotomisierung von Opfer- und Täterschaft aufzugeben, da diese mit einer vereinfachenden Zuordnung von Schuld (Täter) und Unschuld (Opfer) einhergeht. Vielmehr sollte sich die Erkenntnis durchsetzen, dass im Kreislauf der Gewalt die Rollen von Opfer und Tätern von den Beteiligten wechselweise besetzt werden.

Die Entwicklung des EGE und die Aufnahme dieses Interviewverfahrens in die Routinediagnostik der Fachklinik, die während der ersten Behandlungswochen durchgeführt wird, haben dazu geführt, dass insgesamt eine Sensibilisierung für die Gewaltthematik stattgefunden hat und dass das Thema Gewalt die ihm zustehende Bedeutsamkeit gemäß der Untersuchungsergebnisse erhält.

\section{Literatur}

${ }^{1}$ Draijer N. Die Rolle von sexuellem Missbrauch in der Ätiologie psychischer Störungen bei Frauen. System Familie 1990; 3: 59-73

2 Baer A. Ueber die Trunksucht, ihre Folgen und ihre Bekämpfung. In: v. Leyden E, Klemperer F (Hrsg). Die Deutsche Klinik am Eingange des zwanzigsten Jahrhunderts in akademischen Vorlesungen. VI. Band, 2. Abteilung: Geisteskrankheiten. Berlin: Urban \& Schwarzenberg, 1906: 225-296

${ }^{3}$ Huss M. Alcoholismus chronicus. Stockholm: C. E. Fritze, 1852

4 Pernanen K. Alcohol in Human Violence. New York, 1991

${ }^{5}$ Martin SE, Bachman R. The relationship of alcohol to injury in assault cases. In: Galanter M (Hrsg). Recent developments in alcoholism. Vol. 13. Alcohol and violence. New York: Plenum Press, 1997: 42 - 56

${ }^{6}$ Moss HB, Tarter RE. Substance Abuse, Aggression, and Violence. What Are the Connections? The American Journal on Addictions 1993; 2 : $149-160$

${ }^{7}$ Rice RD. The economic cost of alcohol abuse and alcohol dependence: 1990. Alcohol Health and Research World 1993; 17: 10-18

${ }^{8}$ Klein M. Gewaltverhalten unter Alkoholeinfluss: Bestandsaufnahme, Zusammenhänge, Perspektiven. In: Deutsche Hauptstelle gegen die Suchtgefahren (Hrsg). Jahrbuch Sucht 96. Geesthacht, Neuland, 1995: $53-68$

${ }^{9}$ Klein M. Antisoziales Verhalten, Antisoziale Persönlichkeitsstörung und Alkoholismus. Suchttherapie 2000; 1: 21-26

${ }^{10}$ Bushman BJ, Cooper HM. Effects of Alcohol on Human Aggression: An Integrative Research Review. Psychological Bulletin 1990; 107: $341-354$

${ }^{11}$ Gustafson R. Alcohol and aggression. Journal of Offender Rehabilitation 1994; $21: 41-80$

12 Pihl RO, Peterson JB, Lau MA. A biosocial model of the alcohol-aggression relationship. Journal of Studies on Alcohol 1993; 54 (Supplement 11): $128-139$
${ }^{13}$ Murdoch D, Pihl RO, Ross D. Alcohol and crimes of violence: Present issues. The International Journal of the Addictions 1990; 25: $1065-1081$

${ }^{14}$ Straus MA, Gelles RJ (eds). Physical violence in American families. New Brunswick: Transaction Publishers, 1990

${ }^{15}$ Caetano R, Schafer J, Cunradi CB. Alcohol-Related Intimate Partner Violence among White, Black and Hispanic Couples in the United States. Alcohol Research \& Health 2001; 25 (1): 58-65

${ }^{16}$ Klein M, Zobel M. Prävention und Frühintervention bei Kindern aus suchtbelasteten Familien - Ergebnisse einer Modellstudie. In: Zobel M (Hrsg). Wenn Eltern zu viel trinken. Risiken und Chancen für die Kinder. Bonn: Psychiatrie-Verlag, 2001: 90-104

${ }^{17}$ Rose SM, Peabody CG, Stratigeas B. Responding to hidden abuse: A role for social work in reforming mental health systems. Social Work 1991; 36: $408-413$

${ }^{18}$ Widom C, Hiller-Sturmhöfel S. Alcohol Abuse as a Risk Factor for and Consequences of Child Abuse. Alcohol Research \& Health 2001; 25: No.1

${ }^{19}$ Wetzels P. Gewalterfahrungen in der Kindheit. Sexueller Missbrauch, körperliche Misshandlung und deren langfristige Konsequenzen. Baden-Baden: Nomos, 1997

20 Briere J, Runtz M. Differential adult symptomatology associated with three types of child abuse histories. Child Abuse \& Neglect 1990; 14 : $357-364$

${ }^{21}$ Breslau N, Davis GC, Andreski P et al. Traumatic events and posttraumatic stress disorder in an urban population of young adults. Archives of General Psychiatry 1991; 48: 216-222

22 Kessler RC, Sonnega A, Bromet E et al. Posttraumatic stress disorder in the National Comorbidity Survey. Archives of General Psychiatry 1995; $52: 1048-1060$

${ }^{23}$ Kemmner C, Zemlin U, Dornbusch P. Erfassung von Gewalterfahrungen - EGE. Ein halbstrukturiertes Interviewverfahren. Fachklinik Wilhelmsheim: unveröffentlichtes Manuskript, 2001

${ }^{24}$ Straus MA. The conflict tactics scales and its critics: an evaluation and new data on validity and reliability. In: Straus MA, Gelles RJ (Hrsg). Physical violence in American families. New Brunswick: Transaction Publishers, 1990: 49-73

${ }^{25}$ Klein M. Conflict Tactics Scale - D. Deutsche Forschungsversion. Köln: unveröffentlichtes Manuskript, 2002

${ }^{26}$ Henkel D, Zemlin U, Dornbusch P. Analyse rückfallbeeinflussender Bedingungen bei arbeitslosen Alkoholabhängigen (ARA-Projekt) Teil I. Einführung in die Thematik, Projektziele, Untersuchungsanlage und Ergebnisse zu Beginn der Suchttherapie. Sucht aktuell 2003; 2: $5-14$

${ }^{27}$ Windle M, Windle RC. Scheidt DM, Miller GB. Physical and sexual abuse and associated mental disorders among alcoholic inpatients. American Journal of Psychiatry 1995; 152: $1322-1328$

${ }^{28}$ Krinsley KE, Brief DJ, Weathers FW et al. Problems associated with childhood trauma in substance abusers. Poster presented at the annual meeting of the Association for Advancement of Behavior Therapy. San Diego, CA, 1994

${ }^{29}$ Quinten C, Klein M. Langzeitentwicklung von Kindern aus suchtbelasteten Familien. In: Fachverband Sucht e.V (Hrsg). Suchtbehandlungen - Entscheidungen und Notwendigkeiten. Geesthacht: Neuland, 1999: 235-243

${ }^{30}$ Follette VM, Polusny MA, Bechtle AE et al. Cumulative Trauma: The Impact of Child Sexual Abuse, Adult Sexual Assault, and Spouse Abuse. Journal of Traumatic Stress 1996; 9 (1): 25 - 35

31 Dutton MA. Empowering and healing the battered woman: A model for assessment and intervention. New York: Springer, 1992

32 Beg D, Quinten C. Gewalterfahrung von SuchtpatientInnen - Eine empirische Untersuchung zum Ausmaß und zu den wahrgenommenen psychischen Folgen zürückliegender Gewalterfahrungen. Praxis Klinische Verhaltensmedizin und Rehabilitation 2000; 50: 86-92

${ }^{33}$ Langeland W, Hartgers C. Child sexual and physical abuse and alcoholism: a review. Journal of Studies on Alcoholism 1998; 59: 336 - 348

${ }^{34}$ Bussmann KD. Changes in family sanctioning styles and the impact of abolishing corporal punishment. In: Frehsee D, Horn W, Bussmann KD (Hrsg). Family violence against children a challenge for society. Berlin: De Gruyter, 1996: 39-61 